\title{
ERRATUM
}

\section{Light-activated chemical probing of nucleobase solvent accessibility inside cells}

Chao Feng, Dalen Chan, Jojo Joseph, Mikko Muuronen, William H Coldren, Nan Dai, Ivan R Corrêa Jr, Filipp Furche, Christopher M Hadad \& Robert C Spitale

Nat. Chem. Biol.; doi:10.1038/nchembio.2548; published online 15 January 2018; corrected online 22 January 2018

In the version of this article initially published online, the submission date was incorrectly stated as 21 June 2016 . The correct date is 15 June 2017. The error has been corrected in the PDF and HTML versions of this article. 